DOI: $10.20472 /$ IAC.2020.054.009

\author{
JOSE LUIS DANIEL \\ Texas A\&M University-Kingsville, United States \\ RUTH CHATELAIN-JARDON \\ Texas A\&M University-Kingsville, United States \\ ZHUOFAN ZHANG \\ Texas A\&M University-Kingsville, United States
}

\title{
A STUDY OF WORK AND LIFE VALUES IN THE US: DO SUBCULTURES EXIST?
}

\begin{abstract}
:
While culture has been studied extensively at the national level, the concept needs further analysis at the regional level. The purpose of this research is to study subcultures in different states in the United States. Subculture is being measured using a scale of work and life values. Amazon Mechanical Turk is being used as a tool for collecting data, so far responses from 13 states have been obtained. The sample size covers employees from different industries, and it will be utilized and analyzed using factor analysis and analysis of variance. It is expected that there will be cultural differences in terms of work and life values in some of the sates. Moreover, it also expected that there will be clusters of states with similar work and life values. The findings could provide a guideline to human resource managers about workers' behaviors, remunerations, communication and motivation in different states in the US. Also, this study contributes to academia by expanding the study of subcultures in the US. The country is an interesting scenario because regions such as Illinois, California, New York, Texas, Boston, and Florida could present unique characteristics in terms of work and life values
\end{abstract}

\section{Keywords:}

Subcultures, values, regions

JEL Classification: M15, M16, Y90 